\title{
AVALIAÇÃO DE UM CENTRO DE ATENÇÃO PSICOSSOCIAL BRASILEIRO ${ }^{1}$
}

\author{
EVALUATION OF A BRAZILIAN PSYCHOSOCIAL \\ ATTENTION CENTER
}

\section{EVALUACIÓN DE UN CENTRO DE ATENCIÓN PSICOSOCIAL BRASILEÑO}

\author{
JaCó Fernando SCHNEIDER* \\ Marcio Wagner Camatta ${ }^{* *}$ \\ Cíntia Nasi ${ }^{* * *}$ \\ Angélica Nickel Adamolit*** \\ Luciane Prado Kantorski ${ }^{* * * * *}$
}

\begin{abstract}
RESUMO
O objetivo deste trabalho foi avaliar um Centro de Atenção Psicossocial de Porto Alegre/Brasil. Foi conduzido segundo a modalidade de pesquisa qualitativa de forma a apreender a dinâmica do serviço, a forma como os atores interagem e os sentidos por eles construídos em relação à sua prática, por meio da Avaliação de Quarta Geração. A coleta dos dados ocorreu nos meses de outubro e novembro de 2006. Foram realizadas observações e entrevistas com usuários, familiares e membros da equipe de saúde. Os dados foram agrupados e discutidos segundo as temáticas: atividades terapêuticas, equipe, plasticidade, tecnologias leves, ambiência, inserção da família, políticas públicas, rede de serviços de saúde e sociedade. Com esta avaliação, pensamos ter contribuído para a discussão com os grupos de interesse - família, equipe de saúde e usuários no que tange à reestruturação dos Centros de Atenção Psicossocial.
\end{abstract}

Palavras chave: Saúde mental, serviços de saúde mental, enfermagem, avaliação.

\begin{abstract}
The objective of this study was to evaluate a Psychosocial Attencion Center in Porto Alegre/Brazil. A qualitative evaluation was carried through with the proposal to apprehend the dynamics of the service, the manners how the actors interact and how the sense are constructed by those people, in relation to their practice, through the Fourth Generation Evaluation Data collection was made in the period of 2006 between October and November. We use the observation and interviews with users, family and team. The data were grouped and discussed under

\footnotetext{
${ }^{1}$ Trabalho vinculado ao projeto de pesquisa “Avaliação dos Centros de Atenção Psicossocial da Região Sul do Brasil” - Edital Ministério de Ciência e Tecnologia - CNPq / Ministério da Saúde - SCTIE-DECIT/CT - Saúde 07/2005.

* Enfermeiro. Doutor em Enfermagem. Docente do Departamento de Assistência e Orientação Profissional da Escola de Enfermagem da Universidade Federal do Rio Grande do Sul - UFRGS. Endereço: Rua São Manoel, 963, Santa Cecília, Porto Alegre, Brasil. CEP: 90620-110. E-mail: jaco_schneider@uol.com.br.

${ }^{*}$ Enfermeiro. Mestre em Enfermagem. Doutorando do Programa de Pós-Graduação em Enfermagem da Escola de Enfermagem da Universidade Federal do Rio Grande do Sul - UFRGS, Porto Alegre, Brasil. E-mail: mcamatta@terra.com.br

${ }^{* * *}$ Enfermeira. Mestranda do Programa de Pós-Graduação em Enfermagem da Escola de Enfermagem da Universidade Federal do Rio Grande do Sul - UFRGS, Porto Alegre, Brasil. E-mail: cintianasi@yahoo.com.br.

${ }^{* * * *}$ Educadora Física. Mestranda do Programa de Pós-Graduação em Educação Física Escola Superior de Educação Física da Universidade Federal de Pelotas - UFPel, Pelotas, Brasil. E-mail: Likanickel@gmail.

${ }_{* * * * *}$ Enfermeira. Doutora em Enfermagem. Docente da Escola de Enfermagem da Universidade Federal de Pelotas - UFPel, Pelotas, Brasil. Apoio CNPq. E-mail: kantorski@uol.com.br
} 
the theme: therapeutic activities, team, plasticity, soft technology, environment, integration of the family, public policy, the network of health services and society. We hope to have contributed to the discussion with interest groups in family, team and users to the restructuring of the Psychosocial Attencion Centers.

Key words: Mental health, mental health services, nursing, evaluation.

\section{RESUMEN}

El objetivo de este estudio fue evaluar un Centro de Atención Psicosocial de Porto Alegre/Brasil. Se hizo una pesquisa cualitativa para aprender la dinámica del servicio, la forma como los actores interactúan y los significados que ellos construyen sobre su práctica. La recolección de datos se realizó en el periodo de octubre y noviembre de 2006. Como métodos, se utilizó: la observación y entrevistas con usuarios, familiares y equipo. Los datos fueron agrupados y discutidos según el tema: actividades terapéuticas, equipo, plasticidad, tecnología liviana, medioambiente, inserción de la familia, las políticas públicas, la red de servicios de salud y sociedad. Con esta evaluación se espera haber colaborado a la discusión con grupos de interés, familia, equipo y usuarios para la reestructuración de los Centros de Atención Psicosocial.

Palabras clave: Salud mental, servicios de salud mental, enfermería, evaluación.

Fecha recepción: 09/07/08 Fecha aceptación: 17/06/09

\section{INTRODUÇÃO}

Considerando os Centros de Atenção Psicossocial (CAPS) como serviços substitutivos estratégicos ao hospital psiquiátrico e importantes para o processo de consolidação da reforma psiquiátrica no contexto brasileiro, torna-se relevante a avaliação desses serviços de saúde mental.

Para tanto, por meio de um projeto de pesquisa financiado pelo Conselho Nacional de Pesquisa $(\mathrm{CNPq})$, intitulado Avaliação dos Centros de Atenção Psicossocial da Região Sul do Brasil (CAPSUL), foi realizada a avaliação de CAPS da Região Sul do Brasil (Paraná, Santa Catarina e Rio Grande do Sul). O CAPSUL constitui-se em um estudo quantitativo e qualitativo, privilegiando diferentes dimensões do objeto da avaliação.

Nesse artigo apresentaremos os resultados da etapa qualitativa do projeto, em um dos campos estudados. Esta etapa da pesquisa se constituiu em uma avaliação construtivista, responsiva, com abordagem hermenêuticadialética, tendo como proposta apreender a dinâmica do serviço, a forma como os atores interagem, sejam eles profissionais, usuários do serviço e seus familiares e os sentidos que são construídos pelos mesmos em relação às suas vivências.

O CAPS que foi objeto dessa investigação é denominado Centro de Atenção Integral à Saúde (CAIS) Mental Centro, localizado na cidade de Porto Alegre, estado do Rio Grande do Sul, Brasil. Foi criado no ano de 1996, de acordo com as diretrizes estabelecidas pela Portaria № 224 de 29 de janeiro de 1992, a qual regulamenta todos os serviços de saúde mental no país. Com as mudanças dos parâmetros desta portaria, o serviço readequouse à Portaria $\mathrm{N}^{\circ} 336$, de 19 de fevereiro de 2002, sendo definido, portanto, como CAPS II, considerando seu porte/complexidade e abrangência populacional (1).

Esses serviços constituem-se em espaços protegidos, funcionando em regime de turnos, com o atendimento prestado por membros de uma equipe multiprofissional, em que os usuários integram-se a diferentes modalidades de atendimento durante o dia, retornando, à noite, aos cuidados de seus familiares, em suas casas ou para instituições que os estejam abrigando. 
O CAPS é um dispositivo institucional típico do modo psicossocial, por considerar os fatores políticos e biopsicosocioculturais em que os meios básicos de tratamento formam um conjunto de dispositivos de reintegração sociocultural. Além de considerar o sujeito como principal participante do seu tratamento, contempla os contextos familiar e social como importantes agentes de mudança (2).

Tal modalidade de serviço destina-se, prioritariamente, ao atendimento de sujeitos com transtornos mentais severos e persistentes, em sua área territorial, nas modalidades de tratamento intensivo, semi-intensivo e não-intensivo (1).

Como já referido, o objetivo desta pesquisa foi avaliar um Centro de Atenção Psicossocial de Porto Alegre/Brasil.

\section{MATERIAL E MÉTODO}

O processo teórico-metodológico dessa etapa da pesquisa, de natureza qualitativo, foi norteado pela Avaliação de Quarta Geração (3), tendo, como instrumentos de coleta de informações, a observação e a entrevista com os atores envolvidos - profissionais, usuários e familiares, constituindo-se nos grupos de interesse.

A Avaliação de Quarta Geração é implementada por meio dos pressupostos metodológicos do paradigma construtivista. Trata-se de uma avaliação responsiva em que as reivindicações, preocupações e questões dos grupos de interesse servem como foco organizacional (3).

A coleta de dados ocorreu de outubro e novembro de 2006, sendo os grupos de interesse compostos por 13 usuários, 13 familiares e 13 membros da equipe do CAPS em estudo. O critério adotado para a escolha do número dos participantes ocorreu em função de entrevistarmos pelo menos um membro de cada categoria profissional, totalizando 13 profissionais. Adotamos o mesmo número de usuários e familiares para compor esses outros grupos de interesse.

Como critérios de inclusão dos usuários selecionamos aqueles que frequentassem o serviço há mais de um ano, que tivessem boas condições de comunicação, e que já tivessem freqüentado o serviço na modalidade intensiva. Em relação aos familiares, optamos por aqueles inseridos no serviço e também por aqueles que não participavam da dinâmica do mesmo.

Como instrumentos de coleta de dados foram utilizados observação e entrevistas com os grupos de interesse. Foram utilizadas aproximadamente $150 \mathrm{~h}$ em campo de pesquisa por cada um dos quatro pesquisadores envolvidos, totalizando $600 \mathrm{~h}$; o cotidiano do CAPS, foi observado e vivenciado, captandose o maior número de detalhes deste dispositivo de saúde.

As questões utilizadas nas entrevistas foram sobre o atendimento no CAPS, os problemas identificados no CAPS e sobre a política de saúde mental do município. As entrevistas foram gravadas e transcritas na íntegra. Para garantir o anonimato dos envolvidos neste processo de avaliação os mesmos foram identificados como: Usuários (U), Familiares (F), Equipe (E) e Observadores $(\mathrm{O})$.

A avaliação de serviços de saúde tem como seu objeto privilegiado a ação social organizada com vista às mudanças. Entendemos que a proposta da quarta geração, ao negociar resultados da avaliação com todos os interessados, vem ao encontro desta nova visão da ação do homem que propõe o diálogo entre sujeitos de direito. O envolvimento dos grupos de interesse tem tanto o objetivo de buscar questões mais pertinentes ao contexto do serviço e que tenham significado para eles, como o de fortalecer e aprimorar a capacidade de ação desses grupos (4).

Foi utilizado o círculo hermenêutico dialético (3), que é hermenêutico devido ao seu caráter interpretativo e dialético por implicar 
comparação e contraste de diferentes pontos de vista objetivando um espaço de interlocução dos sujeitos e, quando possível, alcançar um consenso (5).

Como primeiro passo do Círculo, foi realizada uma entrevista, na qual foi solicitado ao primeiro entrevistado que discorresse sobre o atendimento e os problemas por ele identificados no trabalho e sobre a política de saúde mental. Imediatamente após cada entrevista foi realizada uma análise prévia dos depoimentos de forma a identificar questões acerca da avaliação para apreciação do próximo entrevistado, além das questões inicias propostas para o primeiro. Desta maneira, a partir do segundo entrevistado, todos os outros eram convidados a responder as três questões inicias e a opinar a respeito das construções que foram surgindo ao longo das análises das entrevistas de cada sujeito do estudo, em seus respectivos grupos de interesse.

A partir de uma análise prévia dos dados coletados, organizamos núcleos temáticos os quais foram apresentados aos grupos de interesse para negociação, compondo eixos prioritários da avaliação.

Os aspectos éticos foram preservados, respeitando-se a legislação brasileira que trata de pesquisas envolvendo seres humanos do Conselho Nacional de Saúde. O projeto de pesquisa teve parecer aprovado pelo Comitê de Ética em Pesquisa da Universidade Federal de Pelotas, sendo garantido sigilo e anonimato dos sujeitos da pesquisa por meio da utilização do Termo de Consentimento Livre e Esclarecido.

\section{RESULTADOS E DISCUSSÕES}

A análise do processo avaliativo do CAPS foi realizada tendo por base Marcadores Internos, de atividades como suporte terapêutico, equipe, plasticidade do serviço, tecnologias leves, ambiência, inserção da família; e Marcadores Externos, de gestão/ políticas públicas, articulação com a rede de serviços de saúde, e sociedade/ inclusão/ preconceito, que serão descritos na perspectiva dos grupos de interesse e da observação realizada pelos pesquisadores.

Ao analisarmos a estrutura, a ambiência foi um dos fatores estudados no serviço, sobretudo quanto ao espaço, que visa a confortabilidade, privacidade e a individualidade dos sujeitos envolvidos, valorizando o ambiente, garantindo conforto aos usuários e trabalhadores, enquanto um espaço que possibilita o exercício das subjetividades (6).

No período de observação e elaboração dos diários de campo da pesquisa foram coletadas informações que descrevem alguns aspectos que consideramos relevantes, além de algumas falas de usuários, familiares e membros da equipe do serviço, as quais retratam a complexidade da ambiência e, a partir destes dados, encontramos pontos positivos e negativos a ela relacionados. A fala de um observador refere-se a uma das oficinas terapêuticas realizadas, denominada Oficina de Beleza:

O local é agradável, tem materiais específicos para oficina, tem cadeiras de salão para lavar os cabelos, chuveiros adaptados perto dessas cadeiras, pia, 3 espelhos, 1 grande para se enxergarem por inteiro, 2 armários, mesas. Cada um tem sua lixa, aparelho de barbear, pauzinho para limpar unha com seu nome $(\mathrm{O})$.

Na perspectiva dos usuários, a ambiência é evidenciada pela falta de conforto e inadequação da estrutura física e das acomodações, explicitada na fala a seguir: "[...] a gente quer mais conforto [...] Essas cadeiras mesmo, tu senta nessas cadeiras [...] os panos também são duros [...] ainda tem os bancos, mas seria muito melhor se tivesse poltronas"(U4).

Os familiares, ao abordarem aspectos relacionados à ambiência, relatam satisfação com o espaço físico, mas apontam a neces- 
sidade de melhorias na estrutura: "Acho bem interessante (...) está bem dividido assim, está bem legal, supre todas as necessidades. Tem refeitório, tem os consultórios, tem a área de lazer" (F8).

[...] eu acho que poderia ser pintado, eu acho que poderia ter uns bancos melhores, eu acho que poderia ter umas portas melhores, eu acho que poderia ter uma janela melhor, poderia não morrer de calor aqui dentro no verão (F7).

Outro marcador interno estudado compreende as Atividades como suporte terapêutico, em que o usuário pode ou não participar das oficinas, não havendo obrigatoriedade em realizar tarefas. "Eu participo das oficinas mais por causa da doença, tento participar o máximo [...]" (U3).

As oficinas terapêuticas constituem-se em recursos imprescindíveis nos CAPS, já que esse instrumento permite ao usuário sentirse produtivo junto à sociedade, além de possibilitar a expressão simbólica de sentimentos e a elaboração de vivências (7). Contudo, é importante ressaltar que as oficinas não se configurem enquanto entretenimento dos usuários, mas sim que estejam focadas em na sua função terapêutica.

Os familiares expressam satisfação com a realização de oficinas terapêuticas no CAPS, conforme pode ser observado na fala: "Isso ajuda bastante ele, no caso se adaptar ao local e se adaptar às pessoas que estão aqui em tratamento também (...)" (F10).

O plano terapêutico do CAIS Mental preconiza que as oficinas sejam espaços terapêuticos e de convivência, com um fazer atrelado a este espaço, buscando viabilizar e potencializar possibilidades de expressão para o usuário (8).

$\mathrm{Na}$ fala dos usuários, a assembléia geral possibilita a participação e a inserção no serviço e reintegração social: "As assembléias são ótimas, eu não participo sempre, mas ela é muito útil porque várias coisas acontecem em função das assembléias [...]" (U7).

O usuário destaca, como relevante, as atividades culturais realizadas fora do espaço do CAPS, como revela esta fala: "Uma das coisas que eu gostei aqui é irmos no cinema. Então, isso é muito bom, a gente dá uma saída, olha o filme e depois comenta [...]" (U6).

As atividades culturais se configuram em ferramentas importantes de familiarização da sociedade no convívio com a loucura, permitindo aos usuários que ultrapassem os limites da instituição de tratamento e ampliem suas relações (9).

A visita domiciliar e o acompanhamento terapêutico são aspectos destacados como importantes para a retomada do seu cotidiano. "Foram várias vezes na minha casa me visitar quando eu não estava bem; então aquilo para mim é muito gratificante" (U9).

Alguns familiares também consideram importante a visita domiciliar, mesmo não tendo recebido este tipo atendimento: "Eu nunca tive visita domiciliar, mas eu acho que é bom. [...] deve ser bacana, assim, elas irem $\mathrm{e}$, às vezes, acho que as pessoas que não têm condições de irem no CAPS, por isso que elas vão, eu acho" (F10).

Outra atividade realizada pelo CAPS, considerada relevante tanto para os usuários como para os familiares e membros da equipe, é o atendimento e acolhimento aos moradores de rua: "Acho que não há preconceito. Eles ajudam tanto quem tem moradia, quanto o que mora na rua. Acho que o atendimento é igual para todos" (F13).

No entanto, os membros da equipe relatam em seus depoimentos outros elementos relacionados a este tema, como o fato do morador de rua ser um frequentador assíduo do CAPS e a necessidade dos profissionais da equipe estarem dispostos a realizar esta atividade.

Quanto ao processo de trabalho, os profissionais destacam a importância do projeto terapêutico enquanto um elemento norteador do serviço, dando sustentação ao CAPS: "Se não fosse o Projeto Terapêutico não seria 
um CAPS, seria um ambulatório, onde dispensava medicação; eu acho que o Projeto Terapêutico caracteriza o CAPS" (E10).

O projeto terapêutico deve ser caracterizado como individual, mas sem desconsiderar a noção de conjunto, possibilitando, ao longo do processo, o fortalecimento de seu coeficiente de autonomia e de escolha do usuário (10).

Os membros da equipe discorrem sobre o trabalho desenvolvido de forma interdisciplinar, citando algumas atividades realizadas no cotidiano do serviço: "Tem uma equipe interdisciplinar e a gente tenta atender os casos, discutindo as condutas e os encaminhamentos em equipe em duas reuniões: uma reunião de equipe e outra reunião de CAD" (E4). Ao citarem algumas atividades, enfatizam o acolhimento com resultados positivos, destacando os encaminhamentos e a escuta como fundamentais no processo de acolhimento: "[...] eu acho que a equipe de acolhimento está bem, elas avaliam bem e fazem corretamente os encaminhamentos" (E6).

$\mathrm{O}$ acolhimento estabelece relações de proximidade entre o profissional e o usuário. Por meio da escuta e de um espaço de reflexão, abre-se a possibilidade de transformar o usuário em co-responsável por sua saúde, contribuindo na construção de sua autonomia e cidadania (11).

No tocante à equipe, características e organização do trabalho, os usuários relatam que o atendimento é resolutivo e promove a integração entre as pessoas: "[..] a equipe é eficiente, são bem atenciosos. Nem todos são, alguns querem tirar o corpo fora [...]. Mas outros não, outros são bem atenciosos" (U12).

Ao relatarem suas características e a organização do trabalho, os membros da equipe enfocam a importância do planejamento e realização de reuniões na estruturação de um processo de trabalho interdisciplinar, muitas vezes complexo e difícil, como mostra a fala:

[...] eu acho que o trabalho interdiscipli- nar, ele tem se perdido um pouco ao longo do tempo, acho que a gente tenta,está tentando ainda, manter isso e em muitos momentos consegue manter, muito graças as discussões na reunião de equipe (E13).

A interdisciplinaridade surge da necessidade de efetivar e tornar resolutivo os serviços de reabilitação psicossocial, visando que a equipe não perca a noção de conjunto, aspecto importante para a construção de pontes que possibilitem avanços na assistência prestada (12).

Além disso, os membros da equipe expressam a necessidade de capacitação e supervisão do trabalho em equipe como ações que devem andar juntas, de preferência realizadas por alguém de fora do serviço: “[...] eu entendo a supervisão como junto da capacitação, alguém de fora, alguém capacitado, que pudesse estar supervisionando como é que a gente está agindo e nos capacitando para alguns momentos que, às vezes, a gente não sabe como lidar” (E4).

A plasticidade, enquanto capacidade que o serviço tem ou não de acolher as demandas para além das questões relacionadas à doença, estendendo-se para a vida do usuário, pode ser apreendida na seguinte fala: "Bom, eu consegui me aposentar com a ajuda do CAIS; o CAIS me ajudou na minha aposentadoria e isso foi bom pra mim, apesar de eu estar reclamando do meu salário [...]" (U12).

Trazendo elementos sobre plasticidade, os profissionais consideram os seguintes dispositivos como importantes: discussões com outras instituições parceiras, como a Universidade, para atividades de cinema e debate, focalizando o aspecto cultural; o trabalho de geração de renda, incentivando a inserção do usuário no espaço do trabalho; encaminhamento e orientação a pessoas que necessitam de algum atendimento específico, como nos casos de pessoas com uso de substâncias psicoativas. Vejamos a fala: "A gente procura sempre fazer essas parcerias. Estava buscando os atendimentos do grupo dos al- 
coólicos anônimos para uma paciente nossa que também é alcoolista. [...] O que a gente procura é ver o que existe na comunidade que possa dar um suporte para esse indivíduo, para essa família” (E3).

Sobre a inserção da família no processo de trabalho, os usuários evidenciam, em suas falas, que há pouca participação do familiar no CAPS, o que interfere e prejudica no tratamento: "Eu achava que tinha que vir alguém da família da gente conviver com o grupo de CAD pelo menos um dia, acho que isso ajudaria bastante" (U3).

Os familiares reafirmam essas falas ao destacarem a necessidade de maior atenção à família, com momentos de escuta do familiar do usuário: “[...] que eles dessem mais atenção ao familiar, por que o familiar sofre junto, acho que sofre muito mais que o paciente, sofre muito mais. Acho que tem que ter a hora do familiar poder falar [...]" (F5).

Os profissionais da equipe expressam dificuldades para inserir a família no contexto do serviço, indo ao encontro das falas anteriores dos usuários e seus familiares; no entanto, consideram essa inserção importante. "A gente ainda tem, enquanto equipe, dificuldade de prestar um atendimento familiar, uma hora o familiar atrapalha, outra hora o familiar tem que ser responsabilizado, outra hora o familiar é um aliado para pensar o tratamento" [...] (E13).

Envolver a família no tratamento de forma a ampará-la, apoiá-la e orientá-la na difícil tarefa de ser cuidadora do usuário deve fazer parte do trabalho de um serviço de saúde mental como o CAPS, tendo em vista que, frequentemente, a família permanece desassistida em seu cotidiano (13).

Além disso, a participação da família no atendimento dos sujeitos em sofrimento psíquico torna-se essencial, por ser esta a primeira célula social da qual o sujeito participa, em que são construídos seus primeiros laços de afetividade. Nesse contexto, a família passa a ser reconhecida como fundamental nos moldes de atenção psicossocial (14).
No resultado da atenção psicossocial, os usuários veem o atendimento como resolutivo, pois promove integração entre as pessoas em um ambiente familiar, ajudando o usuário a se organizar: "E aqui dentro me serviu para eu reorganizar minha vida. E não só a mim, um monte de gente chega aqui de um jeito, depois fica de outro, bem diferente" (U2).

Os resultados da atenção psicossocial descritos pela equipe evidenciam melhorias; no entanto, sinalizam para a necessidade de avanços:

\begin{abstract}
"Eu acho que ao longo dos anos tem melhorado muito, mas acho que precisaria avançar, porque acho que em termos de diagnosticar, de buscar inserir o sujeito no tratamento, no equipamento de assistência social, até acho que a gente consegue um bom sucesso nisso [...]” (E13).
\end{abstract}

Para que ocorram resultados, o modo psicossocial deve estar inserido no campo sócio-cultural, possibilitando, assim, uma atuação com os diversos segmentos da sociedade, constituindo-se em uma estratégia de atuação em saúde mental enquanto substitutivo ao modo asilar de assistência à pessoa em sofrimento psíquico (15).

Quanto às políticas públicas, os usuários, em seus depoimentos, referem-se à necessidade de um CAPS 24 h. "O CAPS 24 horas seria importante [...] acho que um atendimento 24 horas seria ótimo [...] o CAIS tem sido muito bom para mim, mas de noite eu ficava sozinha e se eu me sinto mal de noite como é que eu fico" (U9).

Ao discorrerem sobre questões relacionadas à política de medicação, os membros da equipe destacam que havia essa distribuição no serviço, o que não ocorre mais. No entanto, essas mudanças dificultaram o acesso dos usuários à medicação, afetando diretamente o trabalho no CAPS: "Quando a farmácia saiu daqui, rapidamente a gente viu quanta dificuldade que tem do paciente ir em busca 
essa medicação [...] falta medicação" (E8).

Ainda com relação às políticas públicas, gestão e articulação da rede de saúde, os familiares destacam a necessidade de um maior número de profissionais e da ampliação de CAPS na cidade: "[...] precisaria aumentar, ter mais profissionais para poder atender um número maior de pessoas. [...] as pessoas precisam e a psiquiatria está muito cara para quem não pode. [...] ter mais CAPS nos bairros" (F1).

Os membros da equipe, ao falarem sobre estas questões, relatam dificuldades de encaminhamento para outros setores da saúde, considerando a estrutura da rede: "Conhecendo a rede nos dá um certo trabalho de conseguir endereçar esse paciente para outro lugar [...] estamos tendo um número maior de pacientes encaminhados para cá que poderiam estar em outros lugares" (E4).

Para que seja eficaz, a rede de atenção em saúde mental deve estar organizada de forma efetiva e eficiente, tanto nos aspectos técnicos como nos burocráticos, além de considerar a sensibilidade de forma que a equipe e serviços possam acompanhar as pessoas e assistí-las em suas necessidades, possibilitando suporte à organização do serviço (16).

Os membros da equipe revelam ainda insegurança em relação à continuidade do serviço, devido às mudanças ocorridas na secretaria responsável pela gestão da política municipal de saúde mental: "[...] eu acho que a interlocução com a Secretaria da Saúde, é bem complicada, acho que nunca foi fácil para nós, tanto é a dificuldade da gente tocar o nosso serviço, o nosso trabalho" (E3).

Emerge também uma preocupação no que tange à inserção do CAPS em uma política municipal que parece não investir nesta modalidade terapêutica. Vejamos uma fala nesse sentido: "Por mais que a gente se esforce e mostre um pouco do trabalho, não existe um investimento que avalie nada nessa direção" (E4).

Em contraponto, a equipe busca uma política intersetorial, destacando-se a parceria com outras instituições como algo fundamental para o fortalecimento e consolidação da rede:

Eu acho que a questão das parcerias é muito importante para nós. A gente faz parceria com as unidades básicas de saúde, os PSFs [...] é esse atendimento que muitas vezes a gente espera que as unidades básicas também tenham, que possam contar conosco, que possam pensar, que nos convide a pensar junto, não simplesmente a gente pensar por todos (E3).

Considerando a concepção atual de saúde, situada no cenário do Sistema Único de Saúde (SUS), as práticas de saúde precisam assumir um caráter intersetorial, pois existe uma multiplicidade de variáveis que interferem na produção de saúde.

A falta de interesse político dos gestores e a crônica falta ou ineficiência da aplicação de recursos por parte dos poderes públicos e privados envolvidos com a saúde mental levam a uma constante dificuldade no que concerne à consolidação da reforma psiquiátrica, interferindo nos serviços substitutivos, como é o caso dos CAPS (17).

Em relação ao fenômeno da loucura, os usuários relatam existência de preconceito da sociedade frente ao sofrimento psíquico: "As pessoas julgam a gente na sociedade, as pessoas te chamam de louco [...]. Ninguém gosta de ser chamado de louco" (U3).

Ao pensar na visão da sociedade sobre a loucura, os profissionais alertam para preconceitos ainda existentes, destacando a exclusão das pessoas em sofrimento psíquico: "Acho que as pessoas não sabem o que é a loucura; tem muito preconceito, é muito difícil das pessoas entenderem o que é a loucura e de lidar com isso! Então, eu acho que a exclusão continua"(E6).

Percebe-se que o preconceito existente no momento em que se afirma que as pessoas consideram a loucura como algo estranho, leva à exclusão, ao medo na sociedade, sendo 
a exclusão e o preconceito frutos de algo socialmente construído (18).

Diante dessa situação, deve-se conceder a toda pessoa em sofrimento psíquico o direito de ser tratada e atendida independente de sua condição social, econômica ou política, em um cotidiano que privilegie as subjetividades.

\section{CONSIDERAÇÕES FINAIS}

A avaliação deste Centro de Atenção Psicossocial possibilitou discussão com os grupos de interesse família, membros da equipe e usuários sobre aspectos do serviço. A negociação abriu um espaço para reflexão, quando os participantes se sentiram valorizados por serem sujeitos de uma pesquisa, podendo expressar suas idéias sobre o CAPS.

Pensamos que para os profissionais tenha sido importante avaliar o impacto das suas ações, suas condições de trabalho, a problematização de suas realidades, quando puderam ouvir diferentes opiniões. Consideram importante o planejamento e a realização de reuniões na estruturação do seu trabalho, destacando a importância do projeto terapêutico como um elemento norteador das suas ações no serviço. Embora a equipe verbalize a importância da participação da família no CAPS, reconhece ter dificuldades para inseri-la.

Essa reflexão também foi importante para os usuários do serviço e seus familiares, os quais, por meio de sua participação na pesquisa, puderam opinar, vivenciar experiências distintas, reconhecendo-se integrantes e atuantes no serviço. Os usuários relatam que o CAPS possui uma estrutura física inadequada, embora ofereça um atendimento resolutivo que possibilita integração entre as pessoas por meio de oficinas terapêuticas, visitas domiciliares, atividades culturais e assembléia de usuários e familiares. Apesar de haver possibilidade de participação de fami- liares no serviço, eles sentem-se pouco inseridos no CAPS.

Nesse sentido, esperamos que esse estudo de avaliação de um serviço de saúde mental possa contribuir para a reestruturação dos Centros de Atenção Psicossocial, enquanto um espaço de reabilitação psicossocial, indo ao encontro dos pressupostos e diretrizes da reforma psiquiátrica.

\section{REFERÊNCIAS}

1. Ministério da Saúde (BR). Secretaria de Atenção à Saúde. Legislação em Saúde Mental 1990-2004. 5a ed. Brasília: Ministério da Saúde; 2004.

2. Costa-Rosa A. O modo psicossocial: um paradigma das práticas substitutivas ao modo asilar. In: Amarante P, organizador. Ensaios: subjetividade, saúde mental, sociedade. Rio de Janeiro: Fiocruz; 2006. Pp. 141-68.

3. Guba E, Lincoln Y. Fourth Generation Evaluation. Newbury Park: Sage; 1989.

4. Wetzel C, Kantorski LP. Avaliação de serviços em saúde mental no contexto da reforma psiquiátrica. Texto \& Contexto Enferm. 2004; 13 (4): 593-8.

5. Wetzel C. Avaliação de serviço em saúde mental: a construção de um processo participativo [Doutorado tese]. Ribeirão Preto: Escola de Enfermagem de Ribeirão Preto, Universidade de São Paulo; 2005.

6. Ministério da Saúde (BR). Secretaria de Atenção à Saúde. Núcleo Técnico da Política Nacional de Humanização. Ambiência. 2a ed. Brasília: Ministério da Saúde; 2007.

7. Moll MF. A vida social de pessoas com diagnóstico de esquizofrenia, usuárias de um Centro de Atenção Psicossocial [Dissertação]. Escola de Enfermagem de Ribeirão Preto, Universidade de São Paulo; 2008. 
8. Secretaria Municipal de Saúde (Porto Alegre). Planejamento Estratégico. Cais Mental Centro: Centro de Atenção Psicossocial. Porto Alegre: Secretaria Municipal de Saúde; 2003.

9. Alves DS, Guljor AP. O cuidado em saúde mental. In: Pinheiro R, Mattos RA, Organizadores. Cuidado: as fronteiras da integralidade. Rio de Janeiro: CEPESC/ UERJ/ABRASCO; 2006. Pp. 221-40.

10. Goldberg J. Reabilitação como processo: o Centro de Atenção Psicossocial CAPS. In: Pitta A, organizadora. Reabilitação psicossocial no Brasil. São Paulo: Hucitec; 2001. Pp. 33-47.

11. Coimbra VCC. O acolhimento no centro de atenção psicossocial [Dissertação]. Escola de Enfermagem de Ribeirão Preto, Universidade de São Paulo; 2003.

12. Babinski T, Hirdes A. A Reabilitação psicossocial: a perspectiva de profissionais de centros de atenção psicossocial do Rio Grande do Sul. Texto \& Contexto Enferm. 2004; 13 (4): 568-76.

13. Waidman MAP, Gusmão R. Família e cronicidade da doença mental: dúvidas, curiosidade e relacionamento familiar. Fam. Saúde Desenv. 2001; 3 (2): 154-62.
14. Schneider JF, Camatta MW, Nasi C. O trabalho em um Centro de Atenção Psicossocial: uma análise em Alfred Schütz. Rev Gaúcha Enferm. 2007; 28 (4): 5206.

15. Freire FHM, Ugá MAD, Amarante P. Os Centros de Atenção Psicossocial e o impacto do sistema de financiamento no modelo assistencial. In: Amarante P, organizador. Archivos de Saúde Mental e Atenção Psicossocial 2. Rio de Janeiro: Nau; 2005. Pp. 113-42.

16. Pitta A. Tecendo uma teia de cuidados em saúde mental. In: Venâncio ATA, Cavalcanti MT, organizadores. Saúde Mental: campos, saberes e discursos. Rio de Janeiro: IPUB-CUCA; 2001. Pp. 27782.

17. Oliveira WF, Dorneles P. Patrimônio e ambiente da loucura: a formação do profissional de saúde mental e o diálogo com a vida da cidade. In: Amarante P, organizador. Archivos de Saúde Mental e Atenção Psicossocial 2. Rio de Janeiro: Nau; 2005. Pp. 13-43.

18. Amarante P. O homem e a Serpente: outras histórias para a loucura e a psiquiatria. Rio de Janeiro: Fiocruz; 2003. 